\title{
A roadmap for MERS-CoV research and product development: report from a World Health Organization consultation
}

\author{
Kayvon Modjarrad, Vasee S Moorthy, Peter Ben Embarek, Maria Van Kerkhove, Jerome Kim \& Marie-Paule Kieny \\ As part of the World Health Organization (WHO) R\&D Blueprint initiative, leading stakeholders on Middle East \\ respiratory syndrome coronavirus (MERS-CoV) convened to agree on strategic public-health goals and global priority \\ research activities that are needed to combat MERS-CoV.
}

The emergence and persistence of MERS$\mathrm{CoV}$ as a cause of severe respiratory disease 10 years after the outbreak of severe acute respiratory syndrome coronavirus (SARS-CoV) highlights the need for the rapid development of effective interventions against highly pathogenic human coronaviruses. As MERS-CoV grows in global importance-causing disease and death in more than 1,700 and 600 people, respectively, across 27 countries ${ }^{1}$-research and development (R\&D) efforts to design diagnostic, prophylactic and therapeutic products are gaining momentum. In the aftermath of the 2014-16 Ebola epidemic in West Africa and the current Zika virus outbreak, it has become clear that more strategic investments are needed in the early development of diagnostics, therapeutics and preventives against pathogens of pandemic potential ${ }^{2}$. The ultimate goal is to reduce delays between the identification of a public-health emergency and the deployment of effective medical interventions that will save lives and minimize socioeconomic disruption. Toward this objective, the WHO is developing a blue-

Kayvon Modjarrad is in the US Military HIV Research Program, Walter Reed Army Institute of Research, Silver Spring, Maryland, USA. Vasee S. Moorthy, Peter Ben Embarek and Marie-Paule Kieny are in the World Health Organization, Geneva, Switzerland. Maria Van Kerkhove is at the Institut Pasteur, Center for Global Health, Paris, France. Jerome Kim is at the International Vaccine Institute, Seoul, Republic of Korea.

e-mail: moorthyv@who.int print for emergency $R \& D$ to prevent, or at least mitigate, the impact of infectiousdisease outbreaks. MERS-CoV is one of eight pathogens prioritized in the WHO blueprint (http://www.who.int/medicines/ ebola-treatment/W HO-list-of-topemerging-diseases/en/), and it was selected as a case study to demonstrate how accelerated basic and applied research, as well as product development, could be better supported and coordinated. The WHO therefore convened a consultation of leading experts (Supplementary Note 1) on 10-11 December 2015 to develop a roadmap for MERS-CoV activities as part of the blueprint agenda.

\section{Baseline assessment and epidemiology}

The WHO's assistant director-general for health systems and innovation, Marie-Paule Kieny, opened the consultation by framing the meeting in the context of the broader blueprint for $\mathrm{R} \& \mathrm{D}$ preparedness and emergency response for known priority pathogens. The first author of this report then presented a landscape analysis of MERS-CoV diagnostics, preventives and therapeutics, highlighting the major gaps and advances in ongoing research and product-development activities. This baseline analysis summarized a report that was written for the consultation and that can be accessed on the WHO website (http://www.who.int/csr/research-anddevelopment/mers-landscape.pdf?ua=1). After these overviews, a series of presentations described current knowledge about MERS-CoV epidemiology.

There is already broad consensus within the scientific community that dromedary camels are the main animal reservoir and source of zoonotic transmission to human populations $^{3}$. However, the dynamics of transmission events from dromedaries to humans and between humans are poorly understood. Although studies have demonstrated that individuals with close and frequent contact (i.e., occupationally exposed) with dromedaries are at much higher risk for MERS-CoV infection than the general population ${ }^{4}$, it remains unclear what routes of exposure mediate viral transmission most efficiently. Since the recognized introduction and entrenchment of MERS-CoV in human populations, the majority of MERS-CoV outbreaks have occurred in the nosocomial setting ${ }^{5}$. Although surveillance programs, observational studies and enhanced infection-control systems are now being prioritized and implemented throughout the Middle East, there is still wide variability across the region in the reach of surveillance efforts, the depth of case investigations and adherence to infection-control protocols. Improvements in these efforts will require political will, coordination across multiple sectors within countries at highest risk for future outbreaks, more streamlined communication, engagement with affected communities and cross-validation of diagnostics already in use and in development.

\section{Diagnostic-assay development and standardization}

During the early stages of infection, MERSCoV cannot be clinically distinguished from other types of respiratory infections. Thus, 
Table 1 MERS-CoV monoclonal antibodies in development. Several groups have identified monoclonal antibodies that have at least shown potent neutralization against MERS-CoV, and in some cases, that have protected transgenic mice and NHPs from MERS-CoV disease after viral challenge.

\begin{tabular}{|c|c|c|c|c|}
\hline Institution & Name & Source & Target & R\&D \\
\hline Chinese Academy of Sciences, China & $4 \mathrm{C} 2,2 \mathrm{E} 6$ & RBD-immunized mouse & RBD & Mouse efficacy \\
\hline $\begin{array}{l}\text { Dana-Farber Cancer Institute, USA; } \\
\text { Abviro, USA }\end{array}$ & 3B11 (AV-3) & Human-antibody library & RBD & Mouse and NHP efficacy \\
\hline $\begin{array}{l}\text { New York Blood Center, USA; } \\
\text { Fudan University, China }\end{array}$ & Mersmab1 & S1 immunized mouse & RBD & In vitro \\
\hline Organic Vaccines, USA & m336, m337, m338 & Human-antibody library & RBD & $\begin{array}{l}\text { Mouse, rabbit, and NHP } \\
\text { efficacy }\end{array}$ \\
\hline Regeneron, USA & REGN3048/REGN3051 & Humanized mouse & RBD & Mouse and NHP efficacy \\
\hline Tsinghua University, China & MERS-4, MERS-27 & Human-antibody library & RBD & In vitro \\
\hline
\end{tabular}

S1, spike-domain-containing RBD; S2, spike-domain-containing fusion machinery.

as the current case definition of MERS-CoV infection is based on laboratory confirmation $^{6}$, the development and harmonization of sensitive, specific and easily administered diagnostic assays are crucial to the success of surveillance systems, epidemiologic studies and efficacy assessments in clinical trials. One of the principal challenges of developing useful diagnostic assays is that they are dependent on high levels of virus replication and thus cannot detect infection until several days after viral exposure. During this time, the individual is infectious and poses great risk to others. Speaking to these issues, several participants surveyed the diagnostics in use and commented on their current utility and future viability for both clinical and research purposes.

Nucleic acid-amplification tests (NAATs) are currently the gold standard of MERS-CoV diagnostic platforms ${ }^{7}$. Although these tests have become substantially easier to implement, their performance is still dependent on specimen quality and technician training, because environmental contamination can easily confound accurate interpretation of results. Serologic assays-such as those based on immunofluorescence, immunochromatography, enzyme-linked immunosorbence and live-virus or pseudovirus neutralization-vary in their performance characteristics, but provide benefits over NAATs in the form of easier implementation and more functionally relevant readouts ${ }^{8}$. The performance of any assay, particularly with respect to MERS-CoV, depends on when it is administered during the natural history of disease. A more detailed understanding of the key features of the clinical course of MERS-CoV infection is, therefore, needed to inform the optimization of existing assays and the development of next-generation diagnostics. It was generally agreed that, even in the absence of these data, there is an urgent need for rapid, point-of-care diagnostics for both human and animal populations. The suggestion was made to develop commercially available-or at least adequately validatedsimple, dipstick immunochromatographic assays that are suitable for use on livestock and humans (before confirmatory testing of positive results in humans). It was also recommended that more-advanced diagnostics, such as real-time reverse-transcriptase polymerase chain reaction (rRT-PCR) assays, be developed as part of a multivalent respiratory panel. There is also a need for more coordinated efforts to sequence circulating viruses and to correlate those data with phenotypic outcomes, such as viral fitness, virulence and structure-function relationships of the surface Spike (S) and other MERS-CoV proteins. There was a general call for the development of commercial tests and quality assurance of existing assays, although some of this work has already been started ${ }^{7}$. The validation of diagnostic tests will be essential for the execution and interpretation of epidemiologic studies that can better define viral reservoirs, transmission dynamics and correlates of protection.

\section{Therapeutics}

There are currently no licensed treatments for MERS-CoV. The discovery of antivirals for MERS-CoV has been limited to the repurposing of compounds already licensed or in development for other diseases. Some of the experimental treatments used sporadically during this outbreak are the same as those used for SARS-CoV. As in the SARS-CoV epidemic, however, the use of treatments such as ribavirin, interferons and corticosteroids have yielded little to no clinical benefit, despite showing efficacy in nonhuman primates (NHPs) ${ }^{9}$. High-throughput screens of large libraries have uncovered pharmaceutical agents across several classes that inhibit
MERS-CoV replication in vitro and a few that improve survival in marmoset models ${ }^{10}$. Investigational agents are also being repurposed from other infectious diseases, such as Ebola virus disease, for potential use against MERS-CoV. Data were presented on the antiviral furthest along in development: GS-5734, an adenine analog that incorporates into viral RNA to disrupt replication. It has been shown to protect NHPs from Ebola virus disease and is now advancing through a phase 1 doseescalation trial ${ }^{11}$. So far, its activity against MERS-CoV has been tested only in cell lines.

Antibodies, both monoclonal and polyclonal, have eclipsed antivirals as the focus of MERS-CoV therapeutic R\&D. Initially, convalescent plasma administration, which had been used in other emerging infection outbreaks, was seen as a potentially expeditious and effective means of post-exposure prophylaxis in the setting of cluster outbreaks $^{12}$. A regional protocol was developed, but ultimately could not be implemented, owing to a lack of sufficient convalescent donors $^{13}$. Meanwhile, a different formulation of polyclonal antibodies, derived from a transchromosomal humanized bovine model, has been moving forward in development. Both prophylactic and therapeutic use of this polyclonal preparation significantly reduces viral lung titers in mice that were intranasally transduced with adenoviral vectors that expressed the human MERS-CoV cognate protein receptor, DPP4 (ref. 14). However, it was noted by several experts that therapeutic studies conducted thus far in animals may not be relevant to human outbreaks, given that products are administered only hours after challenge, probably before symptoms in humans would appear.

Among products being researched for preor post-exposure prophylaxis, monoclonal antibodies (mAbs) targeting the receptorbinding domain (RBD) of $S$ are furthest 
Table 2 MERS-CoV vaccine candidates in development. There are five general vaccine platforms in development for MERS-CoV. At the time of this report, all candidates are still in different preclinical stages of development.

\begin{tabular}{|c|c|c|c|c|c|c|c|c|}
\hline \multirow[t]{2}{*}{ Vaccine platform } & \multirow[t]{2}{*}{ Institution } & \multirow[t]{2}{*}{ Product } & \multicolumn{3}{|c|}{ Stage of preclinical development } & \multicolumn{3}{|c|}{ Stage of clinical development } \\
\hline & & & In vitro & Immunogenicity & Efficacy & Phase 1 & Phase 2 & Phase 3 \\
\hline Live attenuated & $\begin{array}{l}\text { Universidad Autonoma } \\
\text { de Madrid, Spain }\end{array}$ & $\begin{array}{l}\text { Recombinant } \\
\text { MERS-CoV }\end{array}$ & & & & & & \\
\hline & $\begin{array}{l}\text { Central South University, } \\
\text { China }\end{array}$ & $\begin{array}{l}\text { RBD fused with } \\
\text { human } \mathrm{Fc}\end{array}$ & & & & & & \\
\hline & Chinese CDC, China & Truncated RBD & & & & & & \\
\hline DNA & $\begin{array}{l}\text { GeneOne Life Sciences, } \\
\text { South Korea }\end{array}$ & Full-length S & & & & & & \\
\hline Prime-boost & $\begin{array}{l}\text { National Institutes } \\
\text { of Health, USA }\end{array}$ & $\begin{array}{l}\text { Full length S DNA } \\
\text { prime, S1 subunit } \\
\text { protein boost }\end{array}$ & & & & & & \\
\hline & University of Oxford, UK & ChAd5 S & & & & & & \\
\hline & $\begin{array}{l}\text { Paul Ehrlich Institute, } \\
\text { Germany }\end{array}$ & Measles S & & & & & & \\
\hline & $\begin{array}{l}\text { Ludwig Maximilian University } \\
\text { of Munich, Germany }\end{array}$ & MVA S & & & & & & \\
\hline
\end{tabular}

Fc, crystallizeable fraction of a human antibody; RBD, receptor-binding domain of the spike glycoprotein; Ad, adenovirus; Ad41, adenovirus serotype 41; MVA, modified vaccinia Ankara virus.

along in the product-development pipeline $\mathrm{e}^{15}$ (Table 1), some of which were presented at the meeting. Although representatives from each of the groups developing MERS-CoV $\mathrm{mAbs}$ presented data on the origin, potency, breadth and animal efficacy of their respective mAbs, some common themes emerged from the session as a whole and from the discussion that followed. Because most of the antibodies that have been developed target the RBD, there is a potential for viral escape from any one $\mathrm{mAb}$. Thus, there should be greater efforts to (i) monitor circulating strains to assess viral evolution; (ii) define and measure phenotypic correlates of viral sequences; (iii) investigate the use of combination $\mathrm{mAbs}$ or polyclonal sera to overcome the potential emergence of therapeutic resistance; and (iv) study the serum of human survivors to better understand the response to natural infection and to develop reference reagents.

\section{Vaccines}

The global will to develop a coronavirus vaccine faded in the aftermath of the SARS$\mathrm{CoV}$ pandemic, but has since gained renewed momentum in the face of the current MERS$\mathrm{CoV}$ outbreak. This session started with a broad overview of vaccine-development pipelines and stressed that most vaccines fail to advance beyond phase 1 testing because of a lack of interest from funders and limited industrial support. In the realm of emerging infectious diseases with suspected or known pandemic potential, governmental agencies and nongovernmental organizations might have a key role in the development of interventions against diseases that do not provide a strong incentive for private-sector investments, but that are still relevant to public health and global security.

There are currently a dozen vaccine candidates in preclinical development (Table 2). Seven of the groups presented their products at the meeting. All developers are basing their immunogen designs on the $S$ surface glycoprotein, the primary target for neutralizing antibodies during natural MERS-CoV infection ${ }^{16}$. Multiple platforms can be used to produce $S$, including but not limited to those presented at the meeting, such as liveattenuated viruses, DNA vectors, protein subunits and viral vectors (i.e., adenovirus, modified vaccinia virus Ankara and measles virus $)^{17}$. Several of the products presented have demonstrated protection in at least one animal model. Although most of the vaccine candidates in the pipeline are being developed for human use, two of the vac- cines have been tested in camels, which, if effective, would interrupt transmission of the virus to humans. A successful example of this "OneHealth" strategy_in which human, animal and environmental concerns are all considered-was described for the vaccine against Hendra virus in horses ${ }^{18}$. One of the lessons learned from the Hendra experience is that preclinical development and animal-model testing in relevant smaller animal models should be extensive before efficacy trials are commenced in larger-animal target populations, such as horses, or in the case of MERS-CoV, dromedary camels. Downselection of vaccine candidates in smaller animals increases the likelihood of success in large animals, which is crucial because the costs are much higher and logistical challenges much greater in the latter models. An additional lesson to take from previous experiences with animal vaccines for human health is to engage affected populations and educate them on the potential benefits and risks of a vaccine for their animals (i.e., camels) and communities.

One of the difficulties facing the development of effective vaccines for MERS-CoV is the absence of an animal model that recapitulates the pathogenesis and natural history of severe human disease. Two presentations and 
a robust discussion centered on this limitation in the field. Several mouse models that are transgenic for the human DPP4 protein receptor have now been developed ${ }^{19}$. Despite their manifestation of clinical disease, data from these transgenic mice might need to be supplemented with that of other, larger animal models for clinical advancement and ultimate licensing. Semi-permissive NHPs have been used as an animal surrogate in vaccineefficacy testing thus far ${ }^{20}$, but it is not clear whether either the rhesus or marmoset NHP models will serve as an accurate proxy for human disease, given that knowledge of the human pathology of MERS-CoV infection is limited to a single autopsy ${ }^{21}$. The development of more relevant animal models requires parallel investigation and elucidation of the virus's pathogenesis in humans. Additionally, there is some concern that a vaccine developed against a new coronavirus may induce antibody-dependent enhancement of infectivity and eosinophilic pulmonary infiltrates, as was observed among mice vaccinated with a virus-like particle (VLP) or killed-inactivated SARS-CoV vaccine ${ }^{22}$. However, none of the vaccines currently being developed for MERS-CoV includes the VLP or killedinactivated platforms.

The roadmap for MERS-CoV R\&D (Supplementary Note 2) will focus on vaccines that are indicated for populations and purposes of priority to the WHO perspective. Three broad indications for vaccination were discussed. The first two indications are for human use. They include a single-dose vaccine to be deployed for individuals at acute risk during outbreaks and a two-dose vaccine to induce durable protection for those at continual risk, such as camel handlers and health-care workers. The third vaccine indication proposed is for dromedaries, particularly juvenile camels, which pose a greater risk than older camels of transmitting virus to humans. The endpoint for a veterinary vaccine is likely to be the reduction or prevention of viral shedding, rather than sterilizing immunity. Despite the great potential for a camel vaccine to interrupt the epidemic, there has been a gap in funding for the development of a camel vaccine from conventional sources that support veterinary vaccines. One reason may be that a MERS$\mathrm{CoV}$ vaccine for camels would be used differently than conventional veterinary vaccines. Usually, animals are vaccinated to prevent illness and death within veterinary populations. In the case of a MERS-CoV camel vaccine campaign, however, the primary intent is to prevent infection and disease in human populations. It will be important to shore up funds for a camel vaccination option, as this may be the fastest developmental and regulatory route toward licensing a product that can prevent human MERS-CoV infections and deaths.

\section{Drafting a research and product development roadmap for MERS-CoV} $\mathrm{R} \& \mathrm{D}$ roadmaps have been used successfully in many sectors in which large-scale, collaborative efforts are required to deliver outcomes related to the innovation and development of new products. In all such processes, it is essential to start by clearly articulating and understanding the goals and markets for these products. High-level priority areas are first identified, after which an agreement on specific activities is decided. It is also crucial to first map out the baseline knowledge gaps and then to develop a strategic plan to address those deficiencies. This also requires an assessment of capacity needs that can support these activities. Project management and implementation structures are subsequently established to pursue agreed-upon activities to reach these goals. An example of this process can be found in the WHO Malaria Vaccine Technology Roadmap, which has culminated in a firstgeneration malaria vaccine and catalyzed the development of second-generation products (http://www.who.int/immunization/topics/ malaria/vaccine_roadmap/en/).

At the meeting, four strategic goals were agreed upon in principle. The first is to establish a surveillance network of coronavirus laboratories as an early warning system to identify circulating species and strains in animal populations, new outbreaks in human populations and emerging strains in all populations. The second is to acquire a better understanding of MERS-CoV pathogenesis, natural history and veterinary and human epidemiology. The third is to develop, manufacture, test, license and use improved diagnostics, preventives and therapeutics that enable the interruption of transmission between humans and from dromedary camels to humans. The fourth, and perhaps most important, is for the global donor community to establish a mechanism that provides a lineof-sight for manufacturers from preclinical proof-of-concept studies to post-licensing procurement of MERS-CoV products, by initiating a public-health financial model for emerging pathogens prioritized by the $\mathrm{WHO}$ blueprint process.

\section{Priority activities to be pursued through the MERS-CoV roadmap}

A series of activities was prioritized and divided into the following categories: (i) basic, translational, epidemiological and social research; (ii) cross-cutting product development that includes refining more relevant animal models, developing reference reagents and designing tools and methods for rational prioritization between products; (iii) improved diagnostics; (iv) therapeutics, mAbs and polyclonal-antibody preparations; (v) vaccines for humans and camels; (vi) capacity development; and (vii) policy and commercialization. These will be further developed in consultation with potential funding stakeholders such as the Biomedical Advanced Research and Development Authority (BARDA), the US National Institutes of Health (NIH), the Wellcome Trust, the European Commission and the International Vaccine Initiative, among others, and with the broader MERS$\mathrm{CoV}$ research community, to include publichealth officials, manufacturers, regulators and product-development partnerships. The draft roadmap was posted for public consultation on WHO's website throughout the month of February 2016 and was finalized in May 2016 (http://www.who.int/ csr/research-and-development/roadmapconsultation/en/).

\section{Summary}

The 2014 Ebola epidemic in West Africa revealed both great potential and pernicious deficiencies within existing mechanisms for rapid medical-product development and deployment. In the aftermath of the epidemic, global health communities coalesced around the realization that a multifaceted plan was required to respond quickly and efficiently to the next outbreak. The WHO is currently developing an $\mathrm{R} \& \mathrm{D}$ blueprint by which such preparation and response can follow, highlighting MERS-CoV as a case study. Although global coordination has resulted in the maturation of the preclinical pipeline for novel interventions for MERS-CoV, products will have to be developed along faster than normal timelines, with greater investments by multiple agencies for development, manufacturing and preclinical and clinical testing, as well as preparations for timely efficacy testing in affected populations if the incidence of disease rises sharply. As the global community takes lessons from the recent Ebola crisis, applies them to the current Zika virus outbreak and prepares for the potential of another regional epidemic or broader pandemic, stakeholders in research and product development on emerging pathogens must set out a sound strategy now for where to best target their investments in anticipation of future outbreaks. The current consultation is a first step toward that end, 
having opened a forum for global dialogue between public-health agencies, scientists, product developers and funders to engage in joint planning of MERS-CoV R\&D activities.

Note: Supplementary Information and Source Data files are available in the online version of the paper.

\section{ACKNOWLEDGMENTS}

We gratefully acknowledge input to the roadmapdevelopment process from all those who attended the WHO consultation in December 2015. A supplementary file lists all those who attended. The views, findings and conclusions within are those of the authors and should not be construed to represent the positions of the US Department of Defense or the World Health Organization.

\section{COMPETING FINANCIAL INTERESTS}

The authors declare competing financial interests:

details are available in the online version of the paper.

1. WHO. http://www.who.int/csr/disease/coronavirus infections/MERS-CoV_summary_update_20140611. pdf (World Health Organization, Geneva, 2014).

2. Cohen, J. Science 351, 16-19 (2016).

3. Ben Embarek, P.K. \& Van Kerkhove, M.D. Wkly. Epidemiol. Rec. 90, 245-250 (2015).

4. Reusken, C.B. et al. Emerg. Infect. Dis. 21, 14221425 (2015).

5. Drosten, C. et al. Clin. Infect. Dis. 60, 369-377 (2015).

6. World Health Organization. (WHO/MERS/LAB/15.1) (World Health Organization, Geneva, 2015).

7. Pas, S.D. et al. J. Clin. Virol. 69, 81-85 (2015).

8. Park, S.W. et al. Euro Surveill. 20, 30042 (2015).

9. Falzarano, D. et al. Nat. Med. 19, 1313-1317 (2013).
10. Dyall, J. et al. Antimicrob. Agents Chemother. 58, 4885-4893 (2014).

11. Warren, T. et al. (IDWeek, San Diego, abstract no. LB-2) (IDWeek, Arlington, Virginia, USA, 2015).

12. Mair-Jenkins, J. et al. Convalescent Plasma Study Group. J. Infect. Dis. 211, 80-90 (2015).

13. Arabi, Y. et al. Springerplus 4, 709 (2015).

14. Zhao, J. et al. Proc. Natl. Acad. Sci. USA 111, 49704975 (2014).

15. Modjarrad, K. J. Virus Erad. 2, 1-4 (2016)

16. Wang, L. et al. Nat. Commun. 6, 7712 (2015).

17. Modjarrad, K. Virus Res. (in the press).

18. Middleton, D. et al. Emerg. Infect. Dis. 20, 372-379 (2014).

19. Agrawal, A.S. et al. J. Virol. 89, 3659-3670 (2015).

20. Falzarano, D. et al. PLoS Pathog. 10, e1004250 (2014).

21. Ng, D.L. et al. Am. J. Pathol. 186, 652-658 (2016).

22. Tseng, C.T. et al. PLoS One 7, e35421 (2012) 